can predict the after scores in Gummerman (1971) very nicely from the before scores. Presumably, this analysis would also be applicable to the data of Egeth \& Smith (1967); however, these authors did not define the master set, M, for their Os, so it is not possible to make the calculations.

\section{REFERENCES}

Birdsall T. G \& Peterson, W. W. Probability of a correct decision in a choice among $m$ alternatives. Electronic Defense Group, University of Michigan, Quarterly Progress Report No. 10, 1954.

Egan, J. P., \& Clarke, F. R. Psychophysics and signal detection In J. B. Sidowski (Ed.), Experimental methods and instrumentation in psychology. New York: McGraw-Hill, 1966. Pp. 211-246.

Egeth, H., \& Smith, E. E. Perceptual selectivity in a visual recognition task. Journal of Experimental Psychology, 1967, 74, 543-549.
Elliott, P. B. Tables of d'. In J. A. Swets (Ed.), Signal detection and recognition by human observers. New York: Wiley, 1964. Pp. 651-684.

Green, D. M., \& Birdsall, T. G. The effect of vocabulary size on articulation score. In J. A. Swets (Ed.), Signal detection and recognition by human observers. New York: Wiley, 1964. recognition

Green, D. M., \& Swets, J. A. Signal detection theory and psychophysics. New York: Wiley, 1966.

Gummerman, K. Selective perception and the number of alternatives. The American Journal of Psychology, 1971, 84 , 173-179.

Lawrence, D. H., \& Coles, G. R. Accuracy of recognition with alternatives before and after the stimulus. Journal of Experimental Psychology, 1954, 47, 208-214.

\section{NOTE}

1. Gummerman, K., and Dykes, J., in preparation.

(Received for publication July 24, 1973.)

\title{
Formation of extinction sets in monkeys
}

\section{ISAAC BEHAR* \\ U. S. Army Medical Research Laboratory, Fort Knox, Ky. 40121}

Discrimination-sophisticated Old World monkeys received 108 two-choice problems, each containing 4 to 8 discrimination trials followed by 4 extinction trials for four animals and by 10 extinction trials for four animals. Following this, they received 108 additional problems with extinction trial length reversed. With 10 extinction trials per problem, an orderly increase in the efficiency of extinction occurred, with one-trial extinction occurring in a large proportion of problems. Virtually no tendency to extinguish occurred with four extinction trials per problem. There was a marked increase in responses to the formerly negative stimulus on the second extinction trials.

According to Harlow, the defining characteristic of a learning set is the change in efficiency with which learning takes place. It is a change from trial-and-error learning to "adapting to a changing environment ... by seeming hypothesis and insight [Harlow, 1949, p. 51]." Learning sets typically are acquired when the animals are trained on an extended series of problems, all of comparable difficulty level, and each for a small number

*Reprint requests should be sent to Isaac Behar, U.S. Army Aeromedical Research Laboratory, Fort Rucker, Ala. 36360. of trials instead of to some criterion of mastery. Using these general operations, the formation of learning sets has been demonstrated for a wide variety of learning tasks, including object discrimination, reversal, oddity, matching to sample, and object alternation.

The present study represents an application of the principles of learning set formation to another aspect of learning performance, namely, that of extinction. While most previous studies of extinction have been concerned with variables that influence the resistance to extinction, this study is concerned with the efficiency or ease with which extinction can occur. Specifically, the purpose was to determine whether monkeys, given a long series of different extinction problems analogous to the paradigm used in the study of learning set formation, would transfer across extinction problems such that they would eventually extinguish in a single trial.

\section{METHOD}

Eight mature monkeys, four rhesus and four sooty mangebeys, all with excellent learning ability as demonstrated in a wide variety of learning tasks, were Ss. Although the specific test histories varied among animals, all had been trained on object-discrimination learning sets; only one had experience with reversal problems. Just prior to the present study, they were given 60 six-trial object-discrimination problems and, based upon that performance, were assigned to either of two groups equated in learning ability, each consisting of two rhesus and two mangabeys. 

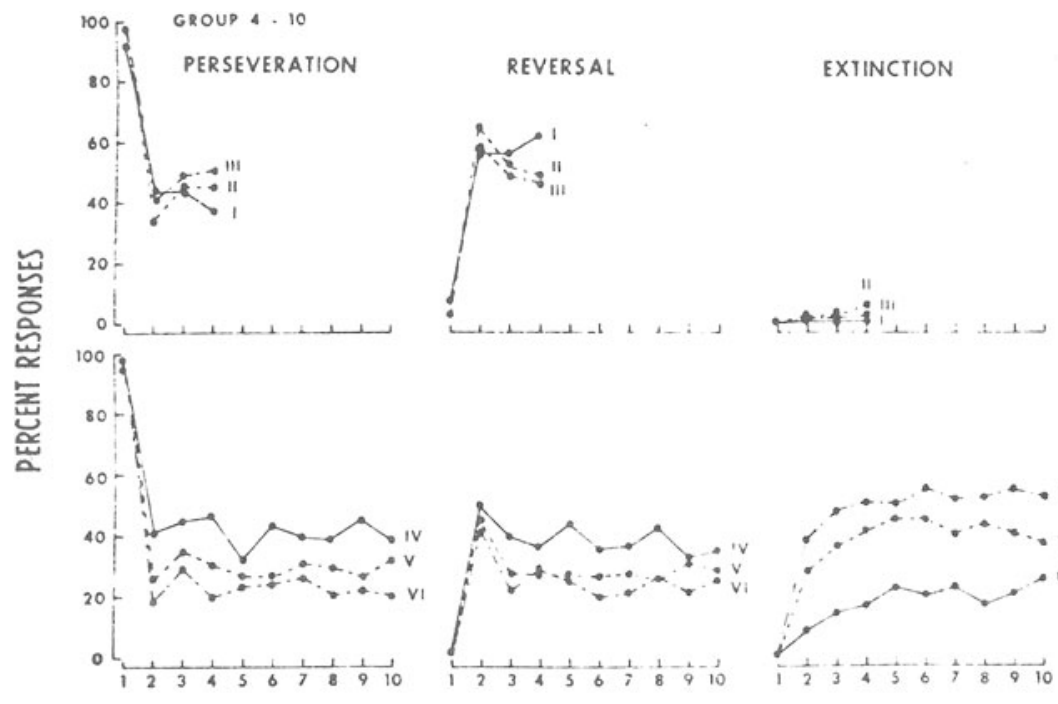

Fig. 1. Percentage of perseveration, reversal, and extinction responses during the extinction trials for Group 4-10. The Roman numerals designate successive blocks of 36 problems.
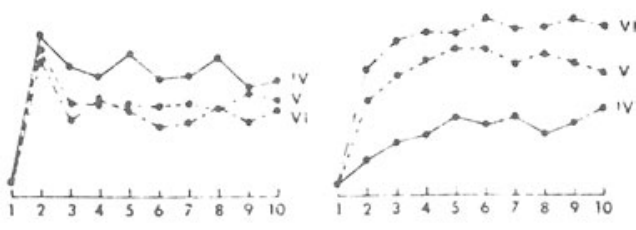

TRIAL

In the present study, all animals were given 216 discrimination problems, each employing unique multidimensional pairs of stimuli. Each problem contained 4, 6, or 8 discrimination trials in which reinforcement was contingent upon correct choice, followed by 4 or 10 extinction trials in which response to neither stimulus was reinforced. Three such problems were given daily over 72 test days. The order of reinforced trial length was counterbalanced among Ss in 6-day blocks. The Ss in Group 4-10 received 4 extinction trials per problem during the first set of 108 problems and 10 extinction trials per problem during the second set of 108 problems. For Group 10-4 the order was reversed.

Testing was conducted in a Wisconsin General Test A'pparatus that was modified so that the raising and lowering of the opaque screen (which separated the $S$ from the test tray) was controlled by a motor-driven lead screw and nut. Timing switches were so located that a Hunter Klockounter was started when the screen cleared the tray and stopped when the $S$ displaced either object.

The procedure for the discrimination trials of each problem consisted of baiting one of the two foodwells with a kernel of corn or a raisin while the opaque screen was in the lowered position. The one-way-vision screen was then lowered between $\mathrm{E}$ and the tray. Next, the opaque screen was raised, permitting the animal to displace one of the objects. Promptly after $S$ responded, the opaque screen was lowered, preventing correction of an incorrect response. The position of the correct object followed a predetermined unsystematic, but counterbalanced, order. The extinction trials followed the 4,6 , or 8 discrimination trials with no deliberate delay and differed only in that reward was absent.

Latency of response, as well as choice, was recorded on every trial. The $\mathrm{S}$ was allowed up to $60 \mathrm{sec}$ to respond during discrimination trials. If the animal failed to respond within that time (a "balk"), the trial was terminated. If the S balked on five successive stimulus presentations, the problem was terminated. On the extinction trials of each problem, the $S$ was allowed up to $30 \mathrm{sec}$ to respond. Failures to respond in $30 \mathrm{sec}$ constituted "extinction responses."

\section{RESULTS \\ Discrimination Performance}

Performance during the discrimination trials was at the high level characteristic of highly sophisticated monkeys. There was no suggestion that discrimination performance was deleteriously affected by the extinction trials; in fact, some improvement occurred for all monkeys between the first and second set of 108 problems, except for one monkey, which dropped from $98.3 \%$ to $98.0 \%$ correct responses (beyond Trial 1). The performance of the two groups remained closely matched (Group 4-10, 92.2\%, Group 10-4, 93.3\% correct). The mean percentage correct for the four rhesus was 91.8, while that for the mangabeys was 93.6.

Only one $S$, rhesus No. 60 , made more than a very rare balk response. This animal, however, balked sufficiently often to terminate 5 problems during the first set of 108, and 6 during the second set.

\section{Extinction Performance}

Performance during the extinction trials is shown in Fig. 1 for Group 4-10 and in Fig. 2 for Group 10-4, in which each curve (Roman numerals) depicts the data for successive blocks of 36 problems. The left panels depict the percent responses to the formerly correct (reinforced) object (perseveration responses) as a function of ordinal extinction trial number. The center panels depict the percent responses to the formerly incorrect object (reversal responses), while the right panels depict the percent of trials in which response to neither object was made within $30 \mathrm{sec}$ (extinction responses). It can be seen that Group 4-10 did not acquire any appreciable tendency to extinguish during the first set of 108 problems, in which the number of extinction trials per problem was 4 . There was, however, a very pronounced increase in reversal responses, with a peak of such responses on Extinction Trial 2. This Trial 2 reversal peak persisted in the second set of 108 problems, during which a characteristic learning set for extinction was acquired. Extinction responses in this set increased significantly over trials $(F=4.09, d f=9 / 27$, $\mathrm{p}<.01)$ and over blocks of 36 problems $(\mathrm{F}=11.96$, df $=2 / 6, \mathrm{p}<.01$ ). The interaction of Trials by Blocks also is significant $(\mathrm{F}=2.48, \mathrm{df}=18 / 54, \mathrm{p}<.01)$. 
Fig. 2. Percentage of perseveration, reversal, and extinction responses during the extinction trials for Group 10-4. The Roman numerals designate successive blocks of 36 problems.
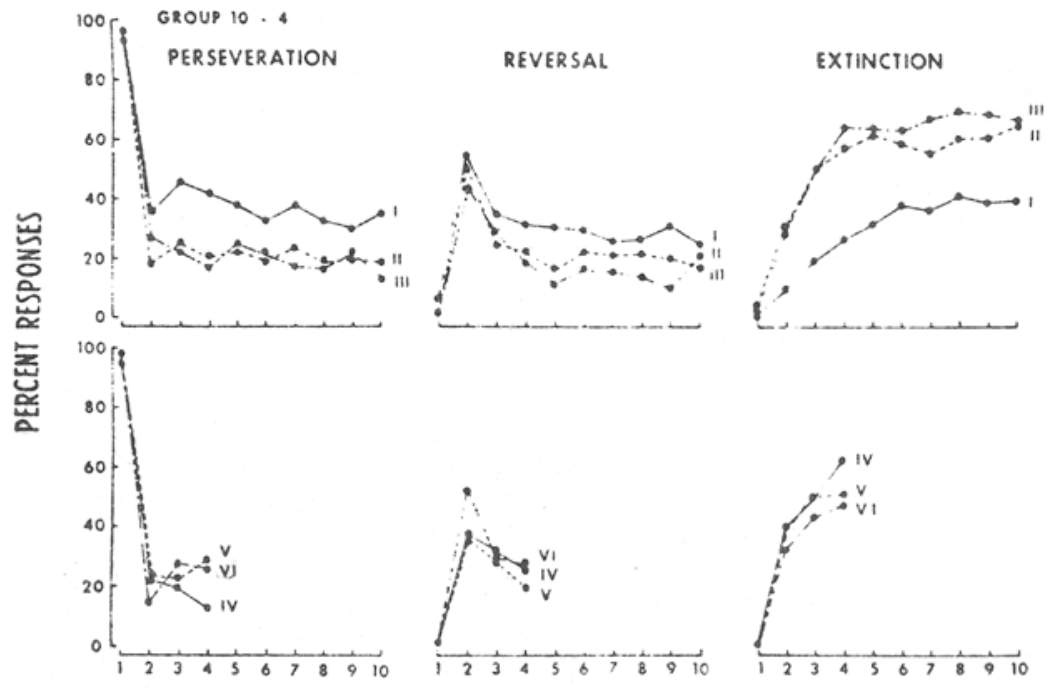

IRIAL
Group 10-4, which had 10 extinction trials per problem during the first set of 108 problems, rapidly acquired an extinction set. Again, both trials $(\mathrm{F}=8.13$, $\mathrm{df}=9 / 27, \mathrm{p}<.001)$ and blocks $(\mathrm{F}=6.63, \mathrm{df}=2 / 6$, $\mathrm{p}<.05)$ are significant, as is the interaction between them $(F=1.97, \mathrm{df}=18 / 54, \mathrm{p}<.05)$. This group also shows a sharp peak of reversal responses on Extinction Trial 2, both in the first and second set of 108 problems. When these Ss were crossed over to 4 extinction trials per problem, there was a small, nonsignificant reduction in extinction responses over the three blocks of 36 problems.

Another analysis of the extinction data evaluated the contribution of discrimination problem length (whether 4,6 , or 8 discrimination trials preceded extinction) and ordinal position (whether first, second, or third problem of the day). For Group 4-10, Condition 10, both length and order were significant variables. The percent extinction responses after 4,6 , and 8 trials, respectively, were $30.55,33.40$, and 35.42 ; that is, the greater the number of discrimination (perhaps, overlearning) trials, the greater the number of extinction responses. For the first, second, and third problem, respectively, the percent extinction responses were $26.81,33.61$, and 38.96.

For Group 10-4, problem length was not significant for either Condition 10 or Condition 4. Ordinal position was significant between the 0.05 and 0.10 level for Condition $10(\mathrm{~F}=3.26, \mathrm{df}=2 / 18)$ and at the 0.05 level for Condition 4. The direction of differences was the same as for Group 4-10, namely, an increase in extinction responses over problems within a session. The percentages were, for Condition 10: $39.74,44.44$, and 47.94; for Condition 4: 31.60, 34.17, and 38.13.

\section{DISCUSSION}

Several previous studies have demonstrated that a single learned habit is both reacquired and extinguished more rapidly after a series of conditionings and extinctions. In fact, Voronin (1962) has determined the number of extinction sessions necessary before extinction occurs after a single nonreinforcement for a variety of animal species. The present data are unique in showing the systematic transfer of extinction which can occur over a long series of different extinction problems, i.e., the formation of an extinction set.

Several characteristics of the extinction set can be noted. First of all, there appears to be a critical number of extinction trials per problem for efficient acquisition; 4 trials clearly are not sufficient, and even 10 trials may be less than optimal. Except for the trials-per-problem variable, the obtained extinction set is characteristic of learning sets generally. The form of the extinction set function initially is approximately linear and later is markedly negatively accelerated. The extinction set asymptotes considerably below $100 \%$, however, but this, in some measure, can be attributed to the very low performance of two animals. However, one-trial extinction did occur fairly often; for the two highest performing Ss it occurred in $67 \%$ and $69 \%$ of the problems.

The high frequency of reversal responses on Extinction Trial 2 is an interesting, if serendipitous, finding and can be attributed to the operation of the "lose-shift" tendency which is learned to a very high level during the acquisition of object-discrimination learning sets. During the discrimination trials, the Ss responded nearly errorlessly (except for Trial 1) to the rewarded object. On the first extinction trial, response to that object no longer was rewarded, bringing into play the lose-shift tendency. As incorrect-object responses also were not rewarded, these responses also declined arThe implication of this pattern of behavior, as it applies to conventional reversal learning sets, is that no new response per se is required for one-trial reversal. Hence, the very rapid acquisition of reversal learning sets by monkeys that had previously acquired discrimination learning sets can be accounted for by the formerly acquired learned tendencies.

\section{REFERENCES}

Harlow, H. F. The formation of learning sets. Psychological Review, 1949, 56, 51-65.

Voronin, L. G. Some results of comparative-physiological investigations of higher nervous activity. Psychological Bulletin, 1962, 59, 161-195.

(Received for publication August 13, 1973.) 